\title{
Injection barrier at metal/organic semiconductor junctions with a Gaussian density-of-states
}

\author{
Sungyeop Jung ${ }^{1}$, Chang-Hyun $\mathrm{Kim}^{1,2}$, Yvan Bonnassieux ${ }^{1}$ and \\ Gilles Horowitz ${ }^{1}$ \\ ${ }^{1}$ LPICM, École polytechnique, CNRS, 91128 Palaiseau, France \\ 2 School of Materials Science and Engineering, Gwangju Institute of Science and Technology, \\ Gwangju 500-712, Republic of Korea
}

E-mail: sungyeop.jung@polytechnique.edu

Received 1 June 2015, revised 20 July 2015

Accepted for publication 27 July 2015

Published 4 September 2015

\begin{abstract}
We physically model the injection characteristics at the metal/organic semiconductor (M/O) junctions with a Gaussian density-of-states (GDOS). By both analytical and numerical modelling, the charge carrier concentrations at the $\mathrm{M} / \mathrm{O}$ junctions in an organic rectifying diode (ORD) are calculated. The results demonstrate a special attention required in the application of the Schottky-Mott rule, which defines the injection barrier (IB) for ideal metal/ semiconductor junctions, to M/O junctions. By systematically changing the width of the GDOS that describes the energetic disorder in the organic semiconductor, we show that the edge of the highest-occupied molecular orbitals (HOMO) should be defined as $\sigma^{2} / 2 k_{\mathrm{B}} T$ higher rather than $2 \sigma$ from the maximum of the HOMO to keep the consistency of the Schottky-Mott rule. A simple analytical expression for the IB is presented which contains the effect of the disorder in facilitating the charge carrier injection. Simulated current density-voltage characteristics of the ORDs are also presented to support the arguments.
\end{abstract}

Keywords: organic semiconductors, solid surfaces and solid-solid interfaces, injection barrier, analytical modelling, finite element method

(Some figures may appear in colour only in the online journal)

\section{Introduction}

Organic semiconductors are characterized by weak intermolecular (or inter-chain) bonds (van der Waals) and small wave function overlap [1]. This results in the stabilization of the energy levels of organic crystals by means of polarization energies so that it differs significantly from the single energy levels of isolated molecules in the gas phase. In addition, compared to the organic crystals, local variation of polarization energies in disordered organic solids, due to different environments (the orientation and distance), leads to a random distribution of energetic states named as a Gaussian density-of-states (GDOS).

Charge carrier injection and transport are crucial in the operation of electronic devices, which depend highly on the electronic structure of the semiconductor. Thus, for organic semiconductors with structural and energetic disorder, the Gaussian disorder model (GDM) was proposed considering a unique electronic structure of organic semiconductors. It establishes that all states are localized and randomly distributed where thermally-assisted tunnelling and trapping (also known as hopping) occurs simultaneously [2]. However, the model focuses on the transport mechanisms, and the role of charge carrier injection has been overlooked in its application to device modelling.

A key parameter governing charge carrier injection is the injection barrier (IB). In the historical point of view, the concept of the IB was firstly provided by Schottky and Mott, as the difference in metal work function and the ionization energy (electron affinity) for holes (electrons), developed for, establishing on the band theory, an ideal metal/semiconductor junction without interaction of any type [3, 4]. 
The theoretical developments on the IB of the metal/ organic semiconductor $(\mathrm{M} / \mathrm{O})$ junctions have dealt with the issues arisen against the Schottky-Mott rule. For example, the existence of an interface dipole was one of the most widely studied issues, which induces the vacuum level mismatch at the junction correspondingly modifying the IB [5]. In addition, the attempts to eliminate the IB by selecting a metal with its work function close to the semiconductor ionization energy (IE) or electron affinity (EA) and their fundamental limit have also been of interest for M/O junctions [6].

Through systematic investigations on these phenomena, it has been shown that the vacuum level mismatch can be incorporated into the effective work function of metal [5]. However, there is still a lack of a physically-based definition of the IB which fully considers the unique features of the GDOS due to divergent definitions of the IE and EA. A fundamental question on how must the IE and EA be determined for organic semiconductors considering the GDOS with indistinct edges has not been clearly answered. Moreover, the consequence of these divergent definitions on device modelling has not been elaborated sufficiently.

The purpose of this paper is to delineate how the IB has been defined and determined for M/O junctions. The physical backgrounds of various definitions are also elaborated distinguishing a charge carrier based definition with transport based definition of the IB. In addition, we present a simple analytical expression for the IB at the M/O junctions with the GDOS, which encompasses the effect of energetic disorder in lowering the IB of such junctions.

\section{Theoretical background}

\subsection{Ideal metal/semiconductor junction}

As shown in figure 1(a), the Schottky-Mott rule defines the IB for an ideal metal/inorganic semiconductor junction [3, 4] as the difference between the metal work function and the semiconductor electron affinity for electrons or the semiconductor ionization energy for holes:

$$
\begin{gathered}
E_{\mathrm{bn}}=W_{\mathrm{m}}-\mathrm{EA}, \\
E_{\mathrm{bp}}=\mathrm{IE}-W_{\mathrm{m}} .
\end{gathered}
$$

This assumes that there are no gap states in semiconductor's forbidden gap and that the conduction and valence band edges are sharp. In addition, the concept of IB in the SchottkyMott rule premises immediate band transport of charge carriers. The latter makes possible a transport-based definition of the IB, which is alternatively written as $E_{\mathrm{bp}}^{\mathrm{t}}=E_{\mathrm{F}}-E_{\mathrm{V}}$ $\left(E_{\mathrm{bn}}^{\mathrm{t}}=E_{\mathrm{C}}-E_{\mathrm{F}}\right)$, as the energy required to inject holes (electrons) from the electrode into the semiconductor at the valence (conduction) band edge.

On the other hand, the IB can also be understood in reference to the charge carrier concentration at the junction. The hole concentration at the metal/semiconductor interface is obtained by integrating the product of the density-of-states function $\delta(E)$ and the Fermi-Dirac distribution for holes $f_{\mathrm{F}-\mathrm{D}}\left(E, E_{\mathrm{F}}\right)=1 /\left[1+\exp \left[-\left(E-E_{\mathrm{F}}\right) / k_{\mathrm{B}} T\right]\right]$ :

$$
p=\int_{-\infty}^{\infty} \delta(E) f_{\mathrm{F}-\mathrm{D}}\left(E, E_{\mathrm{F}}\right) \mathrm{d} E .
$$

For non-degenerate semiconductor, the Fermi-Dirac distribution function is approximated to the Boltzmann distribution function $f_{\mathrm{B}}\left(E, E_{\mathrm{F}}\right)=\exp \left[\left(E-E_{\mathrm{F}}\right) / k_{\mathrm{B}} T\right]$ which simplifies equation (3) into

$$
\begin{aligned}
& p=\int_{-\infty}^{\infty} \delta(E) f_{\mathrm{B}}\left(E, E_{\mathrm{F}}\right) \mathrm{d} E \\
& p=N_{\mathrm{V}} \exp \left(-\frac{E_{\mathrm{F}}-E_{\mathrm{V}}}{k_{\mathrm{B}} T}\right)
\end{aligned}
$$

where $N_{\mathrm{V}}$ is the effective density of state at valence band edge. Now, $E_{\mathrm{bp}}^{\mathrm{c}}=E_{\mathrm{F}}-E_{\mathrm{V}}$ represents a charge carrier based definition of the IB. Note that both approaches result in the same and consistent quantity in the case of ideal metal/semiconductor junction: $E_{\mathrm{bp}}^{\mathrm{t}}=E_{\mathrm{bp}}^{\mathrm{c}}=E_{\mathrm{bp}}$. This can be readily extended for electrons.

Upon the same assumptions, the ideal M/O junctions have been frequently described by the Schottky-Mott rule. In this case, the IB for holes is defined by replacing the valence band edge with the highest-occupied molecular orbitals (HOMO) as $E_{\mathrm{bp}}=E_{\mathrm{F}}-\mathrm{HOMO}$. Similarly, for the IB for electrons, $E_{\mathrm{bn}}=\mathrm{LUMO}-E_{\mathrm{F}}$, where LUMO stands for the lowestunoccupied molecular orbitals.

\subsection{Non-ideal factors}

Although the intuitive picture by Schottky and Mott successfully describes an ideal junction, for a realistic junction, it was observed that the Schottky-Mott rule is not always valid [7]. The primary non-ideal factor is the existence of the vacuum level mismatch $\Delta_{V L}$, which can significantly modify the IB $[8,11]$. Figure $1(\mathrm{~b})$ shows the formation of the vacuum level mismatch. The positive (negative) dipole moment vector $\mu_{\mathrm{D}}$ created by the interface dipole shifts the vacuum level upward (downward), and consequently increases (decreases) the IB for electrons and decreases (increases) the IB for holes by the amount $\Delta_{\mathrm{VL}}$. The interface dipole is often found to be zero either by deliberate passivation with inert buffer layers [9] or by contamination through exposure to atmosphere and/or solvent. However, in general, microscopic charge carrier rearrangements manifested by a variety of chemical and physical process can lead to a non-negligible interface dipole [11-13].

Nevertheless, it is possible to extend the Schottky-Mott rule to a system with a vacuum level mismatch by defining the effective metal work function as the sum of the metal work function and vacuum level mismatch $W_{\mathrm{m}}^{\text {eff }}=W_{\mathrm{m}}+\Delta_{\mathrm{VL}}$ :

$$
\begin{aligned}
& E_{\mathrm{bn}}^{\mathrm{o}}=W_{\mathrm{m}}+\Delta_{\mathrm{VL}}-\mathrm{EA}, \\
& E_{\mathrm{bp}}^{\mathrm{o}}=\mathrm{IE}-\left(W_{\mathrm{m}}+\Delta_{\mathrm{VL}}\right) .
\end{aligned}
$$

Here, the superscript in $E_{\mathrm{b}}^{\mathrm{o}}$ denotes the onset of the ultraviolet photo-emission spectroscopy (UPS) and inverse photoemission spectroscopy (IPES) signals by which the EA and IE 
(a)

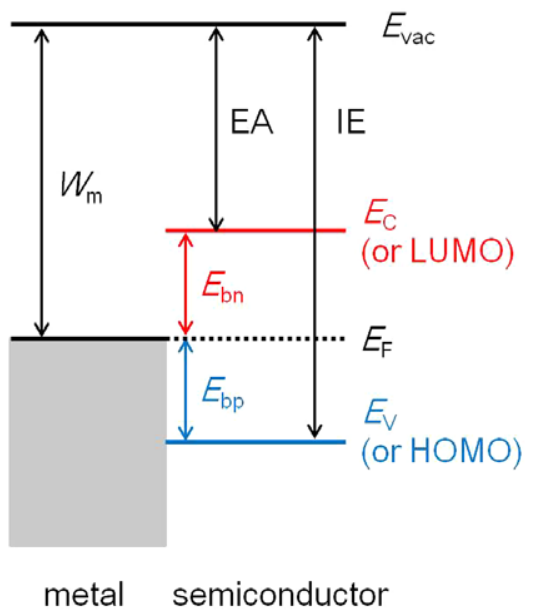

(b)

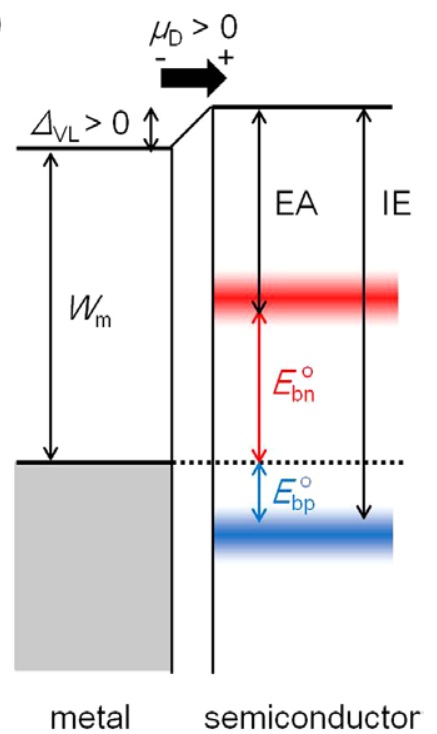

Figure 1. (a) Energy diagram for an ideal metal/semiconductor junction, where $E_{\mathrm{bn}}$ and $E_{\mathrm{bp}}$ denotes the injection barrier (IB) for electrons and holes, respectively. (b) The same for a realistic metal/semiconductor junction with the vacuum level mismatch $\Delta_{\mathrm{VL}}$ and energetic disorder. Here, $E_{\mathrm{b}}^{\mathrm{o}}$ is determined in reference to the onsets of the UPS or IPES signals, which are not specific to the density-of-states model.

are usually determined for both ideal and realistic junctions without specificity to the materials.

The second non-ideal factor is the energetic disorder that are present in solid phase semiconductors to some extent except for a perfect crystal. The energetic disorder illustrated in figure 1(b) originates from the absence of long-range order in the position and direction to a various degree, which requires specific models for the description of the electronic structures discussed in more detail in section 2.3. The presence of the energetic disorder makes difficult a unique determination of the EA and IE, which results in the divergent definition of the EA and IE, and hence the IBs. In case of organic semiconductors, the EA and IE are determined most frequently referring either to the onset $[9,10]$ or the maximum [14] of the HOMO and LUMO. The effect of ambiguity becomes more significant as the metal Fermi level is approaching the indistinct edge and arrives at the fundamental limit called the Fermi level pinning, where the IB does not follows the change in the metal work function. Although often attributed to surface states [15] and metal-induced interface gap states [16, 17], it has been shown that the Fermi level pinning in the organic semiconductor energy gap mainly occurs from electrostatics $[10,18]$. Thus, recalling the considerable effect of the electronic structure on the charge carrier concentration at the junction, a complete understanding of the effect of the energetic disorder on the injection barrier is gaining in importance.

In the following sections, we discuss in detail on the second non-ideal factor. While doing so, we will neglect the existence of the vacuum level mismatch for an exclusive analysis of the effect of energetic disorder.

\subsection{Experimental determination of electronic structures}

The UPS and IPES are the methods of choice to determine the electronic structure of semiconductors. Inspired by the first work on the external photoelectric effect of metals by Einstein [19] and the same of semiconductors by Condon et al [20], Apker et al not only determined the position of the valence band edge but also demonstrated that the density-of-states in the valence band can be experimentally deduced from the energy of photoelectrons [21]. These initial works on photoelectrons support the band theory for crystalline semiconductors providing a link between the existence of the band edge with the sharp cut-off of stopping potential from photo-emission measurement. In addition, experimental determination of the IB based on the Schottky-Mott rule became possible from measured semiconductor IE, EA and metal work function.

For disordered inorganic semiconductors, theoretical models on the electronic structure postulated that the lack of long-range order results in the loss of sharp band edges so that the density of states shows a tail of localized states extending into the energy gap $[22,23]$. Its implication on a transport model, multiple trapping-and-releasing model (MTR), results of a clear distinction between extended states above mobility edge (ME) for band-like transport and localized tail states for charge carrier trapping-and-release [24, 25]. Interpretation of the energy of photoelectrons from amorphous silicon in terms of an exponential tailing of the density-of-states was proposed by Peterson et al [26]. Noting that the transport of charge carriers takes place around the ME, the band edge in the Schottky-Mott model for an ideal junction is replaced with the ME, which is experimentally determined from the onset of the UPS or IPES signals and referred to as the band edge of the same semiconductor in its crystalline phase [27, 28].

The UPS and IPES had been actively utilised for organic semiconductors as well [5], recently paying special attention to their vulnerability to high energy photons and electrons [29]. When the MTR model is applied to disordered organic semiconductors as shown in figure 2(a), the transport based definition of the IB becomes $E_{\mathrm{bp}}^{\mathrm{t}}=E_{\mathrm{F}}-\mathrm{ME}$, which is close 

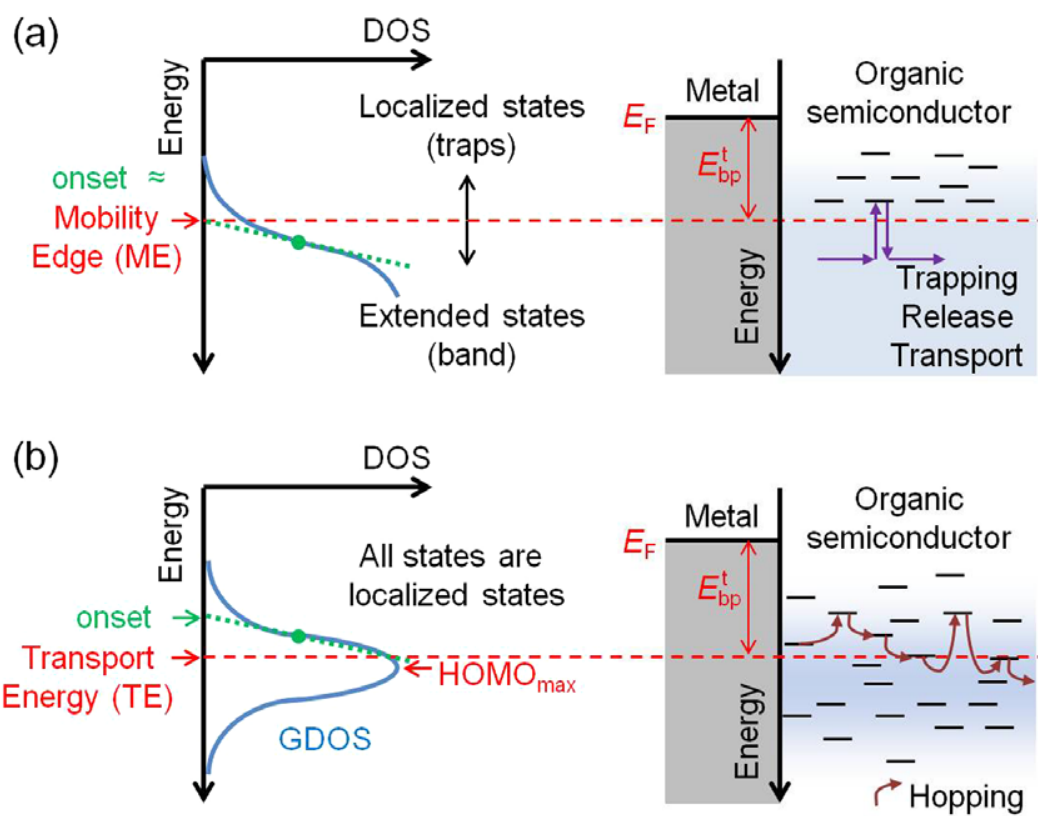

Figure 2. Schematic diagrams for the density-of-states and hole transport mechanism based on the (a) multiple trapping-and-releasing model and (b) Gaussian disorder model. The symbol $E_{\mathrm{bp}}^{\mathrm{t}}$ denotes the transport based definition of the IB.

to the IB determined from the onset of the UPS $E_{\mathrm{bp}}^{\mathrm{t}} \approx E_{\mathrm{bp}}^{\mathrm{o}}$. However, the presence of extended states is unlikely in disordered organic semiconductors. In addition, non-Arrhenius temperature-dependency of mobility is considered as evidence for a Gaussian rather than an exponential density-of-states [30, 31]. In this respect, currently, there is an increasing consensus on interpreting the UPS or IPES signals with a Gaussian function based on an alternative theoretical framework.

\section{Disordered metal/organic semiconductor junction}

In order to describe the characteristic transport of charge carriers in disordered organic semiconductors, the Gaussian Disorder Model (GDM) was proposed, which establishes that all states are randomly distributed and localized where thermally-assisted tunnelling and trapping occurs simultaneously (see figure 2(b)). The density-of-states (DOS) of disordered organic semiconductor is represented by a Gaussian densityof-states function (GDOS), $g(E)$ :

$$
g(E)=\frac{N_{0}}{\sqrt{2 \pi \sigma}} \exp \left\{-\frac{\left(E-\mathrm{HOMO}_{\max }\right)^{2}}{2 \sigma^{2}}\right\},
$$

where $N_{0}$ is the total density-of-states per unit volume and $\mathrm{HOMO}_{\text {max }}$ is the energy at which the DOS is the greatest. In general, we take the density of molecule for $N_{0}$ [32].

In GDM, the hopping transport takes place at a particular energy level called the transport energy (TE), which is indicated in figure 2(b). The essence is that the TE in the GDM is in full analogy with the ME in the MTR model so that the TE can replace the ME [33]. This gives theoretical basis in defining the IB as the energetic difference between the metal Fermi level $E_{F}$ and the TE of semiconductor, $E_{\mathrm{bp}}^{\mathrm{t}}=E_{\mathrm{F}}-$ TE. Numerical analyses predict the position of the

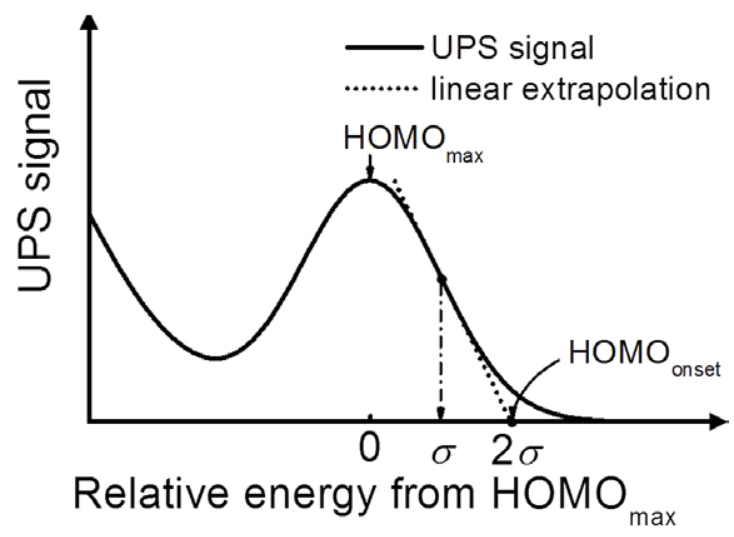

Figure 3. Schematic diagram of the linear extrapolation method used to determine the onset of the highest-occupied molecular orbitals. The linear extrapolation line is drawn at the inflection point at $E=\sigma$ from the $\mathrm{HOMO}_{\max }$. Then, the extrapolation line crosses the base line exactly at $E=2 \sigma$ from the $\mathrm{HOMO}_{\max }$ which is denoted as the $\mathrm{HOMO}_{\text {onset }}$.

TE to be closer to $\mathrm{HOMO}_{\max }$ than to the onset of the HOMO [34]. Thus, the transport based IB can be roughly written as $E_{\mathrm{bp}}^{\mathrm{t}} \approx E_{\mathrm{F}}-\mathrm{HOMO}_{\max }$. Such a definition was used earlier without clear explanation [14]. Note, however, that unlike in the MTR model the onset does not coincide with the TE considering a GDOS so that $E_{\mathrm{bp}}^{\mathrm{t}}$ is different from $E_{\mathrm{bp}}^{\mathrm{o}}$.

This transport based definition of the IB as $E_{\mathrm{bp}}^{\mathrm{t}}=E_{\mathrm{F}}-\mathrm{TE}$ can provide a way of evaluating the injection property. However, it significantly underestimates the effect of gap states in facilitating charge carrier injection. Thus, for a practical reason, the onset of the $\mathrm{HOMO}\left(\mathrm{HOMO}_{\text {onest }}\right)$ is often used in determining the IB in disordered metal/organic semiconductor junction. Figure 3 illustrates the linear extrapolation method (LEM) used to determine $\mathrm{HOMO}_{\text {onset }}$ from the UPS (or IPES) signal assuming a GODS with a width $\sigma$ [6]. In this 

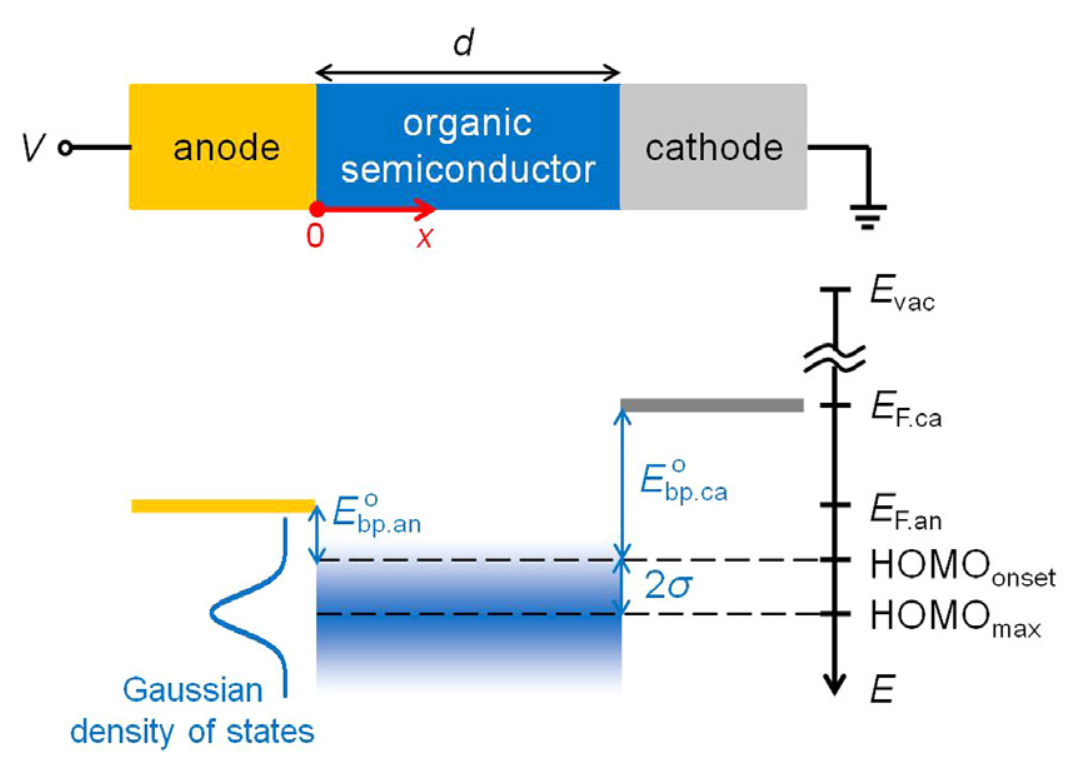

Figure 4. Device structure and energy diagram of an organic rectifying diode. The $\mathrm{HOMO}_{\text {onset }}$ is determined by the method described in figure 2 and fixed while the width of a Gaussian density-of-states $(\sigma)$ is systematically changed as $0.05,0.10$, and $0.15 \mathrm{eV}$. The reference points for the position, potential and energy is indicated.

method, the linear extrapolation line is drawn at the inflection point of which the energetic distance from $\mathrm{HOMO}_{\max }$ is $\sigma$. Then, the extrapolation line crosses the base line exactly at $2 \sigma$ from $\mathrm{HOMO}_{\max }$ which is denoted as the $\mathrm{HOMO}_{\text {onset. }}$. However, it has been little examined whether the onset can substitute the band edge of an ideal semiconductor (see section 2.1).

From now on, as in section 2.1, we discuss on the charge carrier based IB and the correct determination of $\mathrm{HOMO}_{\text {edge }}$ which is equivalent to the band edge of an ideal semiconductor. Under Boltzmann's approximation, the hole concentration at the disordered metal/organic semiconductor junction can be analytically estimated as:

$$
\begin{aligned}
& p=\int_{-\infty}^{\infty} g(E) f_{\mathrm{B}}\left(E, E_{\mathrm{F}}\right) \mathrm{d} E \\
& p=N_{0} \exp \left[-\frac{1}{k_{\mathrm{B}} T}\left\{E_{\mathrm{F}}-\left(\mathrm{HOMO}_{\max }+\frac{\sigma^{2}}{2 k_{\mathrm{B}} T}\right)\right\} .\right.
\end{aligned}
$$

Then, if the numerator on the exponent is considered as the effective charge carrier based IB

$$
E_{\mathrm{bp}}^{\mathrm{eff}}=E_{\mathrm{F}}-\left(\mathrm{HOMO}_{\max }+\frac{\sigma^{2}}{2 k_{\mathrm{B}} T}\right),
$$

it is possible to define the edge of the HOMO from the second and third terms of equation (11) to $\mathrm{HOMO}_{\text {edge }}=\mathrm{HOMO}_{\max }+\sigma^{2} / 2 k_{\mathrm{B}} T$, keeping the SchottkyMott rule consistent for disordered metal/organic semiconductor. This demonstrates that the $\mathrm{HOMO}_{\text {onset }}$ obtained by the LEM can not be used to define a charge carrier based IB. Instead, the $\mathrm{HOMO}_{\text {edge }}$ must be used. In addition, it is now recommended to provide a full set of parameters, the $\mathrm{HOMO}_{\max }$ and the width of a GDOS $\sigma$, from the UPS (or IPES) signal for the analysis of injection characteristics. The consequence of referring to the $\mathrm{HOMO}_{\text {onset }}$ rather than $\mathrm{HOMO}_{\text {edge }}$ is illustrated in rest of this paper.

\section{Analytical modelling}

An organic rectifying diode (ORD) consists of an organic semiconductor layer inserted between a high work function and a low work function electrodes, respectively designated as the anode and cathode. The ORDs under low applied bias (injection-limited regime) will be used here for the analysis of the M/O junction [35].

When analytically modelling ORDs, the following assumptions are made. First, there is no thermally generated free charge carriers inside the organic semiconductor so that it is fully depleted and behaves like a pure dielectric. Second, only holes are injected from both electrodes (hole-only conduction). The result can be straightforwardly extended to the electron-only or bipolar conduction. For the latter instance, it is sufficient to add the electron and hole currents. Third, hole mobility is constant and independent of the hole concentration and electric field. The former is founded on the comparison of the charge carrier density-dependent mobility in diodes and transistors [36], which demonstrated that the mobility is low and less dependent on the charge carrier concentration in diodes where hole concentration is much lower than in transistors. In addition, the latter is established on the comparison of the electric field-dependence of mobility in diodes and transistors [37], which indicates that the mobility is less sensitive to the electric field in diodes. In section 5, the third assumption will be analysed in more detail by comparing the results from analytical and numerical modelling.

Figure 4 shows the device structure and corresponding energy diagram. In the diagram, $E_{\mathrm{bp} . a n}^{\mathrm{o}}$ and $E_{\mathrm{bp} . c \mathrm{c}}^{\mathrm{o}}$ denote the IBs at anode and cathode determined by the LEM (see figure 3). In order to check the validity of the LEM, both analytical and numerical modelling, the width of the GDOS is set at $\sigma=0.05,0.10$ and $0.15 \mathrm{eV}$, while keeping both $E_{\text {bp.an }}^{\mathrm{o}}$ and $E_{\mathrm{bp} . \mathrm{c}}^{\mathrm{o}}$ constant, which is done by correspondingly changing the position of $\mathrm{HOMO}_{\text {max }}$. 


\subsection{Hole distribution}

Under Boltzmann's approximation, equation (12) defines the relationship between the hole concentration and the potential at the position $x$ in the organic semiconductor layer,

$$
p(x)=p_{0} \exp \left\{-\frac{q V(x)}{k_{\mathrm{B}} T}\right\},
$$

where the hole concentrations at the anode $p(0)=p_{0}$ and cathode $p(d)$ are independent of the applied bias:

$$
\begin{aligned}
& p(0)=N_{0} \exp \left(-\frac{E_{\text {bp.an }}^{\text {eff }}}{k_{\mathrm{B}} T}\right), \\
& p(d)=N_{0} \exp \left(-\frac{E_{\text {bp.ca }}^{\text {eff }}}{k_{\mathrm{B}} T}\right) .
\end{aligned}
$$

Here, $\quad E_{\text {bp.an }}^{\text {eff }}=E_{\text {F.an }}-\left(\mathrm{HOMO}_{\max }+\sigma^{2} / 2 k_{\mathrm{B}} T\right) \quad$ and $E_{\mathrm{bp} . \mathrm{ca}}^{\mathrm{eff}}=E_{\mathrm{F} . \mathrm{a}}-\left(\mathrm{HOMO}_{\max }+\sigma^{2} / 2 k_{\mathrm{B}} T\right)$ are the effective injection barrier at the anode and cathode, respectively. Equation (12) indicates that the knowledge of the potential distribution is compulsory to fully determine the hole distribution. Upon assuming the metal-insulator-metal (MIM) model which will be discussed in section 4.2, equation (12) is analytically expressed as

$$
p(x)=p_{0} \exp \left\{\frac{q\left(V-V_{\mathrm{d}}\right)}{k_{\mathrm{B}} T} \frac{x}{d}\right\},
$$

where $V$ is the applied bias at the anode. $V_{\mathrm{d}}$ the diffusion potential with which it holds $q V_{\mathrm{d}}=E_{\mathrm{bp} . \mathrm{ca}}^{\mathrm{eff}}-E_{\mathrm{bp} . \mathrm{an}}^{\mathrm{eff}}$.

\subsection{Potential distribution}

In electrostatics, the relationship between the potential and the charge distribution is given by Poisson's equation:

$$
\frac{\mathrm{d}^{2} V(x)}{\mathrm{d} x^{2}}=-\frac{q p(x)}{\epsilon} .
$$

By using equation (12) and $F(x)=-\mathrm{d} V(x) / \mathrm{d} x$, Poisson's equation develops into

$$
\frac{\mathrm{d} V(x)}{\mathrm{d} x}=\sqrt{-2 \frac{q p_{0}}{\epsilon}\left[\exp \left\{-\frac{q V(x)}{k_{\mathrm{B}} T}\right\}-1\right]+F(0)^{2}},
$$

where $F(x)$ is the electric-field at the position $x$. Note that, because the effect of disorder in lowering the injection barrier is fully contained in $p_{0}$ as a coefficient of the differential equation, equation (17) can be resolved in a equivalent manner to that for crystalline semiconductors reported in [38, 39]. Thus, although a general solution is available, a numerical computation is needed at some point.

As will be shown later, the metal-insulator-metal model is completely valid for ORDs with GDOS as long as the effective injection barrier height is higher than about $0.2 \mathrm{eV}$. This recalls the same behaviour of $\mathrm{M} / \mathrm{O}$ junctions in organic diodes and field-effect transistors in which organic semiconductor was assumed perfectly crystalline $[35,40]$.
In MIM model, the potential distribution is a linear function of the position:

$$
V(x)=-\left(V-V_{\mathrm{d}}\right) \frac{x-d}{d},
$$

where $V_{\mathrm{d}}$ is again the diffusion potential, $V$ the applied bias at the anode and $d$ the thickness of organic semiconductor layer. Note that $V(0)=V-V_{\mathrm{d}}$ and $V(d)=0$, respectively at anode $(x=0)$ and cathode $(x=d)$.

\subsection{Current density-voltage characteristics}

The drift-diffusion model is generally considered as the most appropriate to describe the current in organic diodes, in which the current is defined as the sum of the drift and diffusion currents:

$$
J=q p \mu F-q D \frac{\mathrm{d} p}{\mathrm{~d} x},
$$

where $\mu$ is hole mobility and $D$ diffusion coefficient of holes. In low carrier density limits, i.e. under Boltzmann's approximation, the general relation between $\mu$ and $D$ found in [41] reduces to Einstein relation $D / \mu=k_{\mathrm{B}} T / q$ so that equation (19) can be rewritten as

$$
J=\mu k_{\mathrm{B}} T\left(\frac{p}{k_{\mathrm{B}} T} \frac{\mathrm{dHOMO}_{\max }}{\mathrm{d} x}-\frac{\mathrm{d} p}{\mathrm{~d} x}\right) .
$$

After multiplying by $\exp \left(-\mathrm{HOMO}_{\max } / k_{\mathrm{B}} T\right)$ at both sides as an integrating factor, integration of equation (20) results in

$$
J \int_{0}^{d} \exp \left(-\frac{\mathrm{HOMO}_{\text {max }}}{k_{\mathrm{B}} T}\right) \mathrm{d} x=-\mu k_{\mathrm{B}} T\left[p \exp \left(-\frac{\mathrm{HOMO}_{\max }}{k_{\mathrm{B}} T}\right)\right]_{0}^{d} .
$$

Using $\mathrm{HOMO}_{\max }$ at the cathode as the reference energy, from equation (18), $\mathrm{HOMO}_{\max }(x)$ is expressed as

$$
\mathrm{HOMO}_{\max }(x)=q\left(V-V_{\mathrm{d}}\right) \frac{x-d}{d} .
$$

Then, the boundary conditions for energy are given by

$$
\begin{aligned}
& \operatorname{HOMO}_{\text {max }}(0)=-q\left(V-V_{\mathrm{d}}\right), \\
& \mathrm{HOMO}_{\text {max }}(d)=0 .
\end{aligned}
$$

Combining equations (21) and (22) with the boundary conditions for hole concentration equations (13) and (14) as well as those for energy equations (23) and (24) leads to

$$
J=q \mu p(0) \frac{\exp \left(q V / k_{\mathrm{B}} T\right)-1}{\exp \left(q V_{\mathrm{d}} / k_{\mathrm{B}} T\right)-\exp \left(q V / k_{\mathrm{B}} T\right)} \frac{V_{\mathrm{d}}-V}{d},
$$

where $p(0)$ is the hole concentration at the anode. Using $q V_{\mathrm{d}}=E_{\mathrm{bp} . c \mathrm{a}}^{\mathrm{eff}}-E_{\mathrm{bp} . \mathrm{an}}^{\mathrm{eff}}$, the above can be alternatively written as $J=q \mu p(d) \exp \left(q V_{\mathrm{d}} / k_{\mathrm{B}} T\right) \frac{\exp \left(q V / k_{\mathrm{B}} T\right)-1}{\exp \left(q V_{\mathrm{d}} / k_{\mathrm{B}} T\right)-\exp \left(q V / k_{\mathrm{B}} T\right)} \frac{V_{\mathrm{d}}-V}{d(26)}$.

The main assumption that leads to equations (25) and (26) is that the potential profile remains unchanged when a voltage is applied. This is correlated with the low density limits 
and, as will be shown in the following, restricts its validity to low applied voltages and for relatively little disordered semiconductors.

\section{Numerical modelling}

In this section, we develop on a numerical modelling based on a two-dimensional finite-element method (FEM). Complementary to the analytical modelling, a numerical modelling provides with exact numerical solutions for systems without analytical solution. In this study, a numerical simulator ATLAS from SILVACO is used which resolves a set of coupled Poisson's, drift-diffusion and continuity equations in a self-consistent manner [42]. This makes possible, first, resolution of a system out of thermal equilibrium. Second, the inclusion of a complex dependence of mobility on electric field and charge carrier concentration neglected in analytical modelling though it has profound physical basis. Third, the resolution of non-analytic functions such as the Gauss-Fermi-Dirac integral and general Einstein relation.

The geometric and energetic structures used for numerical modelling are the same as in the analytical modelling, while, for the hole and potential distribution, two conditions out of thermal equilibrium are supplemented $(V=-1$ and $1 \mathrm{~V})$. The simulated hole concentration $p(x)$, potential distribution $V(X)$ and current density-voltage characteristics $J-V$ from the analytical and numerical modellings are compared. Physical and electrical parameters used for modelling are collected in table 1 .

\subsection{Hole distribution}

Figure 5 shows the simulated hole distributions in the organic layer of ORDs with the widths of the GDOS at $\sigma=0.05, \quad 0.10$ and $0.15 \mathrm{eV}$. The hole distributions are numerically simulated under three bias conditions: applied voltage at the anode $V=-1,0$ and $1 \mathrm{~V}$, where the cathode is grounded. Concomitantly, the analytical modelling is conducted exclusively at thermal equilibrium $(V=0)$ by using equations (13)-(15).

It is clearly shown that the hole distribution as well as the hole concentration at the electrodes increase with the increase of $\sigma$ even though $E_{\mathrm{bp} . \text { an }}^{\mathrm{o}}$ and $E_{\mathrm{bp} . c \mathrm{a}}^{\mathrm{o}}$ are fixed at 0.3 and $0.9 \mathrm{eV}$, respectively for the anode and cathode. This demonstrates that, in defining the IB, the $\mathrm{HOMO}_{\text {edge }}$ should not be determined as an energy level at $2 \sigma$ from $\mathrm{HOMO}_{\max }$ towards the Fermi level of the adjacent metal electrode, that is the $\mathrm{HOMO}_{\text {onset }}$ Instead, as shown in equation (11), the $\mathrm{HOMO}_{\text {edge }}$ must be defined as $\mathrm{HOMO}_{\text {edge }}=\mathrm{HOMO}_{\text {max }}+\sigma^{2} / 2 k_{\mathrm{B}} T$ for a correct description of the effect of the energetic disorder, characterized by $\sigma$, on the IB and the hole concentration at the M/O junction. The excellent match between the numerical and analytical modelling results for $\sigma=0.05$ and $0.10 \mathrm{eV}$ at $V=0$ confirms this argument.

In table $2, E_{\mathrm{bp}}^{\mathrm{o}}$ and $E_{\mathrm{bp}}^{\mathrm{eff}}$ are compared for various $\sigma$ at both electrodes. Note that for $\sigma=4 k_{\mathrm{B}} T$ (around $0.1 \mathrm{eV}$ at room temperature), $E_{\mathrm{b}}^{\mathrm{o}}$ and $E_{\mathrm{b}}^{\mathrm{eff}}$ are equal. On the other hand, the
Table 1. Physical and electrical parameters used in the simulation.

\begin{tabular}{lll}
\hline Categories & Parameters & Values \\
\hline $\begin{array}{l}\text { Physical } \\
\text { dimensions }\end{array}$ & Thickness of organic semi- & $200 \mathrm{~nm}$ \\
Anode & conductor layer $(d)$ & \\
Cathode & Fermi level $\left(E_{\mathrm{F}}^{\mathrm{an}}\right)$ & $-4.9 \mathrm{eV}$ \\
Organic & Fermi level $\left(E_{\mathrm{F}}^{\mathrm{ca}}\right)$ & $-4.3 \mathrm{eV}$ \\
semiconductor & HOMO edge & $-5.2 \mathrm{eV}$ \\
& Width of a Gaussian & $0.05,0.10$ and \\
& density-of-states $(\sigma)$ & $0.15 \mathrm{eV}$ \\
& Total density-of-states $\left(N_{0}\right)$ & $10^{21} \mathrm{~cm}^{-3}$ \\
& Hole mobility $(\mu)$ & $0.5, \mathrm{~cm}^{2} \mathrm{~V}^{-1} \mathrm{~s}^{-1}$ \\
& Dielectric constant $(k)$ & 3.6 \\
& Doping concentration $\left(N_{\mathrm{A}}\right)$ & $0 \mathrm{~cm}^{-3}$ \\
& Defect density of states $\left(N_{\mathrm{T}}\right) 0 \mathrm{~cm}^{-2} \mathrm{eV}^{-1}$ \\
\hline
\end{tabular}

Table 2. Effective injection barriers at the anode and cathode.

\begin{tabular}{lllll}
\hline$\sigma(\mathrm{eV})$ & $E_{\text {bp.an }}^{\mathrm{o}}(\mathrm{eV})$ & $E_{\text {bp.an }}^{\text {eff }}(\mathrm{eV})$ & $E_{\text {bp.ca }}^{\mathrm{o}}(\mathrm{eV})$ & $E_{\text {bp.ca }}^{\text {eff }}(\mathrm{eV})$ \\
\hline 0.05 & 0.30 & 0.35 & 0.90 & 0.95 \\
0.10 & 0.30 & 0.31 & 0.90 & 0.91 \\
0.15 & 0.30 & 0.16 & 0.90 & 0.76 \\
\hline
\end{tabular}

LEM underestimates (overestimates) the IB for $\sigma<4 k_{\mathrm{B}} T$ $\left(\sigma>4 k_{\mathrm{B}} T\right)$, respectively. Note also that, for $\sigma=0.15 \mathrm{eV}$, although $E_{\text {bp.an }}^{\mathrm{o}}=0.3 \mathrm{eV}$ foretells the safe applicability of Boltzmann's approximation and MIM model, it is observed that the simulated hole distributions by the numerical and analytical modelling differ at the anode and do not follow an exponential relation with respect to the distance from the anode. In contrast, referring to $E_{\mathrm{bp}}^{\mathrm{eff}}=0.16 \mathrm{eV}$, it is perfectly predictable.

At $V=-1$ and $1 \mathrm{~V}$, numerical modelling shows that simulated hole distributions are not exponential function of the distance from anode. The hole distribution changes abruptly according to the applied bias. On the contrary, the applied bias does not affect the hole concentrations at both electrodes.

\subsection{Potential distribution}

Figure 6 shows the simulated potential in the organic semiconductor layer of ORDs with the width of the GDOS at $\sigma=0.05,0.10$ and $0.15 \mathrm{eV}$, which demonstrates more directly the applicability of the MIM model.

In agreement with the hole distribution, at $V=0$, the potential follows a linear function of the distance for $\sigma=0.05$ and $0.10 \mathrm{eV}$. In the meantime, at $V=0$, the potential deviates from a perfect linear line near the anode for $\sigma=0.15$ eV. Nevertheless, the MIM model seems still fair enough. Remarkably, for all values of $\sigma$, the numerically simulated potential follows a straight line at $V=-1 \mathrm{~V}$, which extends the validity of the MIM model into the reverse bias regime $(V<0)$.

On the other hand, at $V=1 \mathrm{~V}$, holes are dominantly injected from anode that has smaller IB compared to the cathode so that the bending of the potential distribution becomes more 


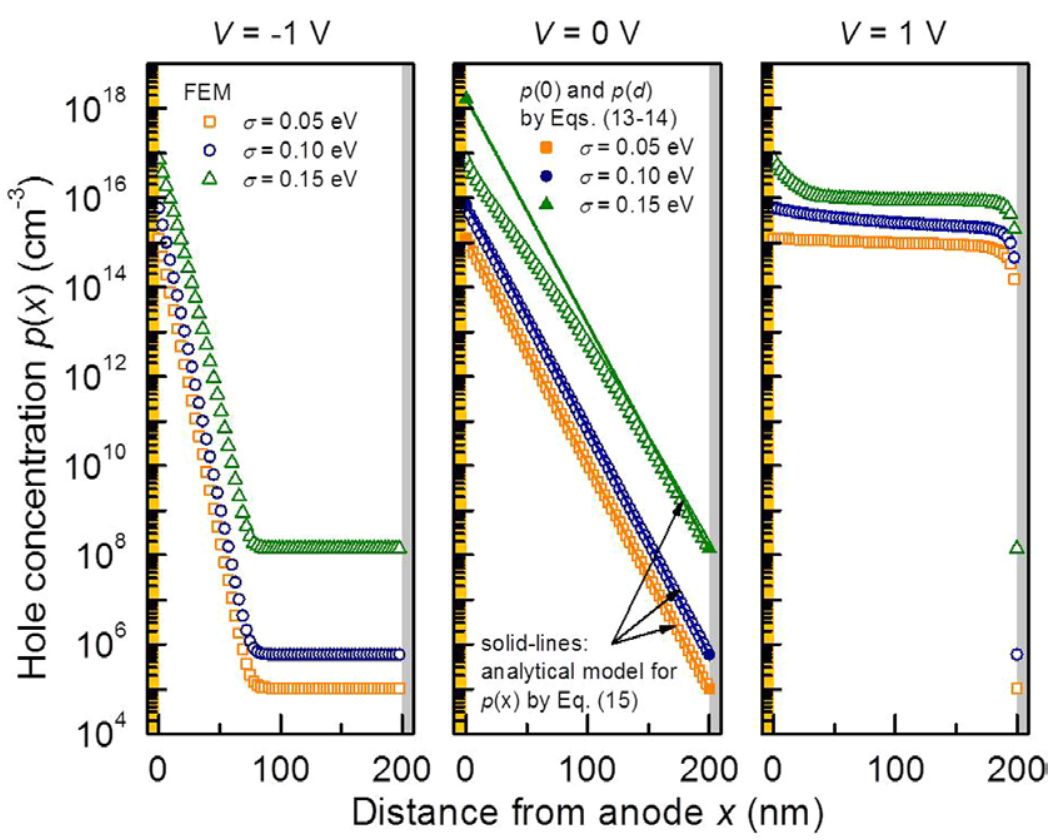

Figure 5. Simulated hole concentration in the organic semiconductor layer at three applied bias conditions: applied voltage $V=-1 \mathrm{~V}$ (left panel), $V=0 \mathrm{~V}$ (centre panel) and $V=1 \mathrm{~V}$ (right panel). The open symbols correspond to the numerical simulation. The solid-lines and filled symbols correspond to the analytical simulation. The width of a Gaussian density-of-states $(\sigma)$ is systematically changed as 0.05 , 0.10 , and $0.15 \mathrm{eV}$.

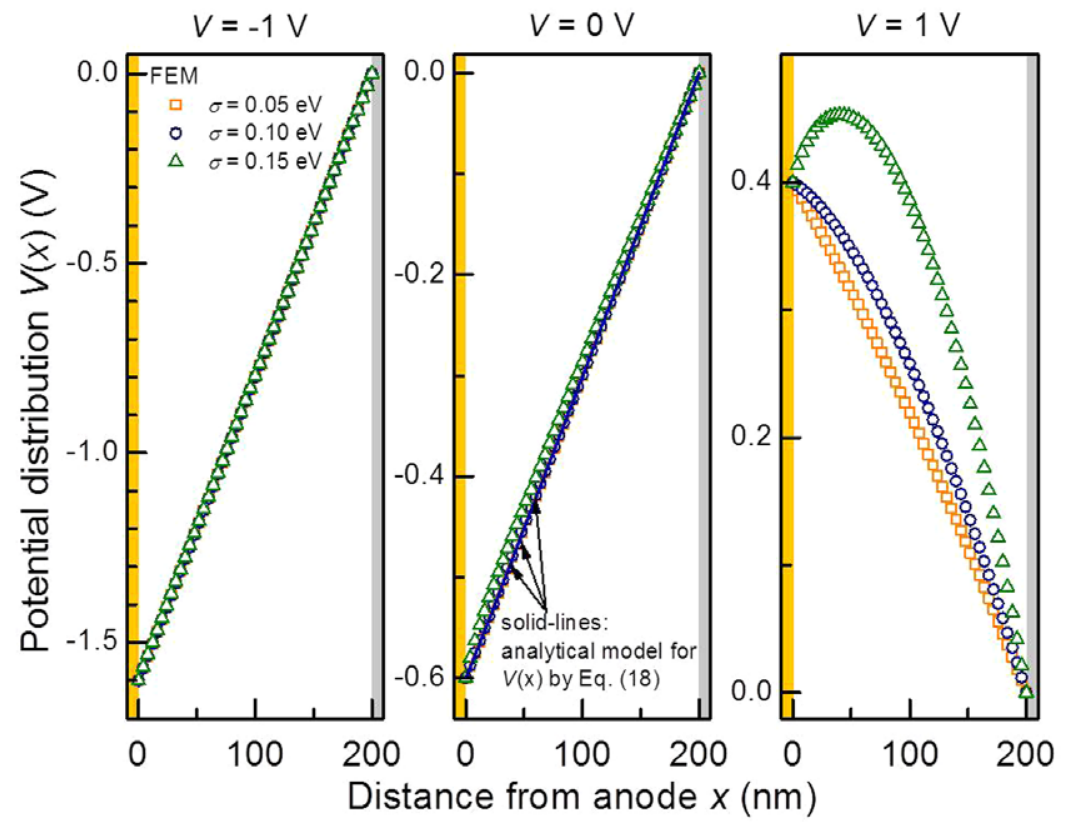

Figure 6. Simulated potential distribution in the organic semiconductor layer at three applied bias conditions: applied voltage $V=-1 \mathrm{~V}$ (left panel), $V=0 \mathrm{~V}$ (centre panel) and $V=1 \mathrm{~V}$ (right panel). The open symbols correspond to the numerical simulation. The solid-lines correspond to the analytical simulation. The width of a Gaussian density-of-states $(\sigma)$ is systematically changed as $0.05,0.10$, and $0.15 \mathrm{eV}$.

significant than at the thermal equilibrium or in reverse bias regime. At variance with what found in the conventional Schottky diode, this originates not from a depletion of charge carriers but from the increase of the charge carrier density. For $\sigma=0.15 \mathrm{eV}$, the space charge region, which is formed near the anode, creates an electric field in the opposite direction to that due to the applied bias. The consequence of this phenomena is going to be illustrated in the next section.

\subsection{Current density-voltage characteristics}

The current density-voltage $J-V$ characteristics of the ORDs with various widths of the GDOS are simulated by numerical and analytical method (see figure 7). Similarly to the ORDs where GDOS is not considered [35], the simulated curves display three distinct regimes: reverse $(V<0)$, injection-limited $\left(0<V<V_{\mathrm{d}}\right)$, and bulk-limited regime $\left(V>V_{\mathrm{d}}\right)$. 


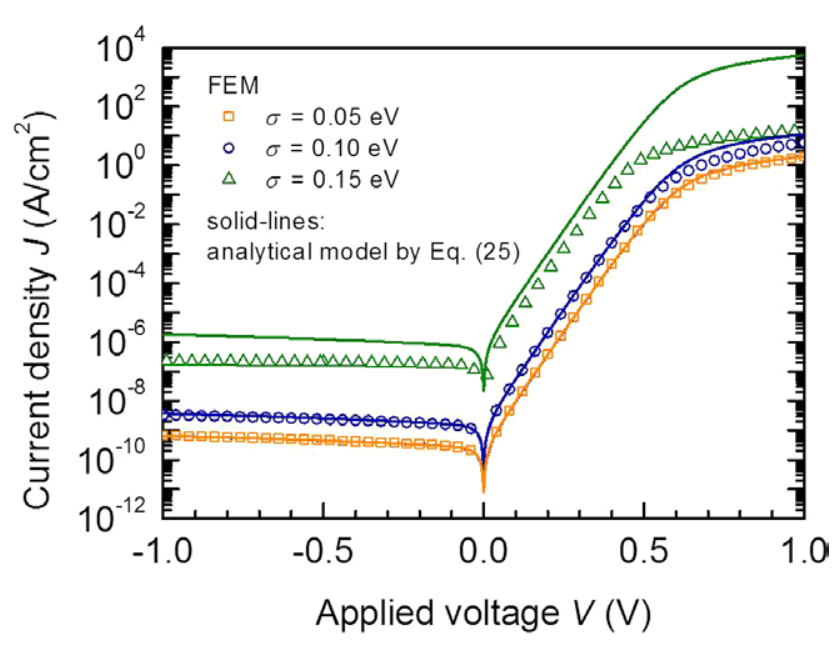

Figure 7. Simulated current density-voltage $J-V$ characteristics of the organic rectifying diodes. The open symbols correspond to the numerical simulation. Solid-lines correspond to the analytical simulation. The width of a Gaussian density-of-states $(\sigma)$ is systematically changed as $0.05,0.10$, and $0.15 \mathrm{eV}$.

In addition, the increase of the current density with the increase of the $\sigma$ brings evidence for an improved charge carrier injection. This is more evident from the result of the numerical modelling, where the mobility is considered as a variable which decreases as disorder increases [37]. Since the current density increases with lowered injection barrier, the result implies that the role of disorder in facilitating charge carrier injection overpasses the concomitant reduction of the mobility.

The difference in the current density from the analytical and numerical modelling in the bulk-limited regime reflects the limits of the MIM model, which becomes more significant when the injection barrier is approaching nil. This originates from the electric field created by the large number of holes injected from anode in the opposite direction to the external electric field. This opposing electric field hinder the movement of holes towards cathode, and hence decreases the current density.

\section{Conclusion}

We have presented a comprehensive analytical and numerical modelling of $\mathrm{M} / \mathrm{O}$ junctions and organic rectifying diodes with a GDOS. In such junctions, we show that the transport and charge carrier based definitions of the IB should be distinguished and that the latter is more suitable for describing the effect of the extended states in facilitating charge carrier injection. In addition, a correct method of determining the position of the $\mathrm{HOMO}_{\text {edge }}$ is presented as the energy level at $\sigma^{2} / 2 k_{\mathrm{B}} T$ rather than $2 \sigma$ above the $\mathrm{HOMO}_{\max }$. Referring to this new definition for the injection barrier, named as the effective injection barrier, a rigorous assessment of a $\mathrm{M} / \mathrm{O}$ junction became possible, including a correct prediction the limit of the metal-insulator-metal model. We expect that our theoretical work provides an insight on understanding the injection process in M/O junctions which is fundamentally different to the metal-inorganic semiconductor junctions and on interpreting the UPS or IPES signals to determine the electronic structure of organic semiconductors.

\section{Acknowledgments}

This work has been funded by Horizon 2020 Marie Skłodowska-Curie Research and Innovation Staff Exchange (RISE) Programme (2015-2019) entitled DOMINO. S Jung acknowledges the financial support by the Vice Presidency for External Relations (DRI) of the École polytechnique through a PhD fellowship.

\section{References}

[1] Silinsh E A 1980 Organic Molecular Crystals 1st ed (Berlin: Springer)

[2] Bässler H 1993 Phys. Status Solidi B 175 15-56

[3] Schottky W J 1939 Z. Phys. 113 367-71

[4] Mott N F 1939 Proc. R. Soc. Lond. A 171 27-38

[5] Amy F, Chan C and Kahn A 2005 Org. Electron. 6 85-91

[6] Hwang J, Wan A and Kahn A 2009 Mater. Sci. Eng. R 64 1-31

[7] Schweikert H 1939 Verhandl. Phys. Ges. 399

[8] Kahn A, Koch N and Gao W 2003 J. Polym. Sci. B: Polym. Phys. 41 2529-48

[9] Fukagawa H, Kera S, Kataoka T, Hosoumi S, Watanabe Y, Kudo K and Ueno N 2007 Adv. Mater. 19 665-8

[10] Oehzelt M, Koch N and Heimel G 2014 Nat. Commun. 5 1-8

[11] Ishii H and Seki K 1997 IEEE Trans. Electron Devices 44 1295-301

[12] Knupfer M and Peisert H 2004 Phys. Status Solidi A 201 1055-74

[13] Koch N and Vollmer A 2006 App. Phys. Lett. 89162107

[14] Ivanco J, Netzer F P and Ramsey M G 2007 J. Appl. Phys. 101103712

[15] Bardeen J 1947 Phys. Rev. 71 717-27

[16] Monch W 1990 Rep. Prog. Phys. 53221

[17] Tung R T 2001 Mater. Sci. Eng. R 35 1-138

[18] Ley L, Smets Y, Pakes C I and Ristein J 2013 Adv. Funct. Mater. 23 794-805

[19] Einstein A 1905 Ann. Phys. 322 132-48

[20] Condon E U 1938 Phys. Rev. 541089

[21] Apker L, Taft E and Dickey J 1948 Phys. Rev. 741462

[22] Mott N F 1967 Adv. Phys. 16 49-144

[23] Cohen M H, Fritzsche H and Ovshinsky S R 1969 Phys. Rev. Lett. 221065

[24] Le Comber P G and Spear W E 1970 Phys. Rev. Lett. 25509

[25] Horowitz G, Hajlaoui M E and Hajlaoui R 2000 J. Appl. Phys. 87 4456-63

[26] Peterson C W, Dinan J H and Fischer T E 1970 Phys. Rev. Lett. 25861

[27] Mott N 1987 J. Phys. C: Solid State Phys. 203075

[28] Tanaka K and Nakayama S-i 2000 J. Optoelectron. Adv. Mater. $25-11$

[29] Yoshida H 2014 Anal. Bioanal. Chem. 406 2231-7

[30] Baranovskii S D, Zvyagin I P, Cordes H, Yamasaki S and Thomas P 2002 Phys. Status Solidi B 230 281-8

[31] Baranovskii S D 2014 Phys. Status Solidi B 251 487-525

[32] Lang D V, Chi X, Siegrist T, Sergent A M and Ramirez A P 2004 Phys. Rev. Lett. 93086802

[33] Baranovskii S D, Rubel O and Thomas P 2005 Thin Solid Films 487 2-7

[34] Baranovskii S D, Faber T, Hensel F and Thomas P 1997 J. Phys.: Condens. Matter 9 2699-706

[35] Kim C H, Yaghmazadeh O, Bonnassieux Y and Horowitz G 2011 J. Appl. Phys. 110093722 
[36] Tanase C C, Meijer E J, Blom P W M and de Leeuw D M 2003 Phys. Rev. Lett. 91216601

[37] Pasveer W, Cottaar J, Tanase C C, Coehoorn R, Bobbert P, Blom P W M, de Leeuw D and Michels M 2005 Phys. Rev. Lett. 94206601

[38] Mott N F and Gurney R W 1940 Electronic Processes in Ionic Crystals 2nd edn (Oxford: Oxford University)
[39] Skinner S M 1955 J. Appl. Phys. 26498

[40] Jung S, Kim C H, Bonnassieux Y and Horowitz G 2015 J. Phys. D: Appl. Phys. 48035106

[41] Ashcroft N W and Mermin N D 1976 Solid State Physics 2nd edn (New York: Holt, Rinehart and Winston)

[42] SILVACO 2014 Deckbuild User's Manual (Santa Clara, CA: SILVACO International) 